\title{
Pricing Scheme of Ocean Carrier for Inbound Container Storage for Assistance of Container Supply Chain Finance
}

\author{
Mingzhu Yu, ${ }^{1}$ Xin Tian, ${ }^{2}$ and Lean $\mathbf{Y u}^{3}$ \\ ${ }^{1}$ Department of Transportation Engineering, College of Civil Engineering, Shenzhen University, Shenzhen 518060, China \\ ${ }^{2}$ Research Center on Fictitious Economy and Data Science, Chinese Academy of Sciences, Beijing 100190, China \\ ${ }^{3}$ School of Economics and Management, Beijing University of Chemical Technology, Beijing 100029, China \\ Correspondence should be addressed to Lean Yu; yulean@amss.ac.cn
}

Received 12 February 2014; Revised 21 May 2014; Accepted 22 May 2014; Published 15 June 2014

Academic Editor: Chuangxia Huang

Copyright (C) 2014 Mingzhu Yu et al. This is an open access article distributed under the Creative Commons Attribution License, which permits unrestricted use, distribution, and reproduction in any medium, provided the original work is properly cited.

\begin{abstract}
The aim of this paper is to investigate the pricing scheme of ocean carrier for inbound container storage so as to assist container supply chain finance. In this paper, how an ocean carrier should set price of inbound container storage to the customer while facing the contract from the container terminal operator is first analyzed. Then, two different contract systems, the free-time contract system which is widely used in practice and the free-space contract system which is newly developed recently, are considered. In the two different contract systems, inbound container storage pricing models are constructed, and accordingly optimal solution approaches for the ocean carrier are provided. For comparison purpose, some numerical experiments for the two different contract systems are conducted to investigate the effects of the container terminal operator's decision on the system outcomes. Numerical experiments show that (1) the carrier is more flexible in the free-space contract system and can receive more profit by using the free-storage-space as a pooling storage system and (2) the free-space contract system benefits both the carrier in profit and the busy terminal in traffic control.
\end{abstract}

\section{Introduction}

The ocean transportation business leads to the scarcity of resources in the container terminal. Therefore, operational efficiency improvement is now one of the main concerns of container terminal operators. The container terminal yard operation plays a critical role in integrated terminal operations because efficiency improvement in the container terminal yard accelerates both the waterside operations and the landside operations. Traditionally, terminal operators try to optimize the schedule of the yard crane and the allocation of containers. Nowadays, they are paying more attention to strategic decisions. Take the inbound container storage for example; if the customer stores an inbound container in a container terminal yard for longer than the so-called freetime-limit, the storage fee will be linear to the container dwell time in the terminal yard and will be charged by the container terminal operator.

Fransoo and Lee [1] find that the operational relationship may not be aligned with the contractual relationship in ocean transportation system. Though there is no direct contractual relationship between the customer and the terminal operator, the operational interaction exists. The inbound container storage reflects the unaligned operational and contractual relationship. Customers store their inbound containers in the container terminal yard. Container terminal operator first charges the storage fee from the ocean carrier, and ocean carrier charges this fee from the customer later. The fees paid to the terminal operator and paid from the customer are not necessarily the same amount. That is, the customer pays the container storage fees to the terminal operator through the ocean carrier.

This paper aims to investigate the pricing scheme of ocean carrier's inbound container storage facing two kinds of contracts from the container terminal operator: the freetime contract and the free-space contract. In the free-time contract setting, the container terminal operator provides a contract consisting of "free-time-limit" and storage fee to the ocean carrier. If the customer's containers stay in the terminal yard for longer than the free-time-limit, each container will 
be charged by container terminal operator per extra day. Given this free-time contract from the terminal operator, the ocean carrier provides a similar contract to the customer with the same free-time-limit value and higher storage fee rate than provided by the container terminal operator. In the free-space contract setting, the container terminal operator provides a contract which includes free-storage-space and storage fee to the ocean carrier. If the storage space occupied by the customers' inbound containers exceeds the free space, the ocean carrier needs to pay the container storage fee for per day per unit container to the container terminal operator. Facing this contract from the container terminal operator, the carrier still provides a free-time contract to the customer. In the free-space contract system, the carrier does not operate the terminal storage yard directly. Hence, it is impossible for the carrier to track the space occupied in the container terminal yard. Therefore, the carrier provides free-time contract rather than free-space contract to the customers.

In the ocean transportation system, the free-time contract system is quite popular. For example, in Hong Kong port and many other Chinese ports, container terminal operators provide the free-time contract to carriers. However, the freespace contract system is newly developed and is not well studied. Some container terminals in South Korea are using the free-space contracts now. In order to fill the gap in the inbound container storage pricing area, we explore the ocean carrier's inbound container storage price schemes with the free-time and free-space contracts from the container terminal operator.

In the context of supply chain finance, solutions and technics are adopted to optimize capital for the buyer and enhanced cash flow for the supplier and to minimize risk in the supply chain. The pricing problems we studied involve three parties: the container terminal operator, the carrier, and the customers, in the container supply chain. We investigate the contractual relations between them, which helps to build the basis for further studies on the container supply chain finance.

\section{Literature Review}

There are extensive studies concerning efficiency improvement of various container terminal operations. Comprehensive reviews could be found in Günther and Kim [2] and Stahlbock and Voß [3]. It is hard to find existing studies on the container storage pricing schemes in supply chain finance. Fransoo and Lee [1] mentioned that extensive research questions need to be answered in the ocean transportation area, where the unaligned contractual and operational relationship in the container supply chain is a critical topic.

de Castilho and Daganzo [4] firstly discussed the effect of the remote warehouse's existence on the customer behavior. In order to minimize the total storage cost, customers between the container terminal and the remote warehouse should be considered. Their paper investigates the pricing issue for the cargo's temporary storage in the container ports under discriminatory and nondiscriminatory schemes. K. H. Kim and K. Y. Kim [5] considered the real practice of the container terminal operator's inbound container storage price, which consists of a free-time-limit and an extra storage fee after the free-time-limit. In their paper, they assumed that a remote container storage yard is located near the terminal. Different models are provided to maximize the container terminal profit which is under a certain customer service constraint or to minimize the total public cost. Lee and $\mathrm{Yu}$ [6] focus on the inbound container storage price competition problem and consider the competition relationship between the container terminal operator and the remote container storage yard operator. The container terminal operator provides the free-time-limit and an off-time storage fee in their pricing scheme, and the remote container yard operator sets the storage price as linear to the container storage time. Both the random container storage time and the sensitive container storage time are considered. They proved the uniqueness and existence of the price equilibrium.

Holguín-Veras and Jara-Díaz [7] studied a joint problem of optimal space allocation as well as storage pricing. The priority price scheme where a different class of containers get different storage charge and neutral price scheme where a unique price is utilized for all types of containers are considered. They extended their research by considering that the container arrival rate is sensitive to the terminal storage charge in Holguín-Veras and Jara-Díaz [8]. Saurí et al. [9] proposed a new import container storage pricing model which has a flat storage fee (a nonzero constant) before the freetime-limit.

Our paper contributes to the literature by considering the unaligned situation in the ocean transportation system. Different from the traditional study, we focus on the pricing scheme of the ocean carrier rather than the container terminal operator.

\section{Model Formulation}

In this paper, an inbound container storage pricing problem, which includes one carrier, one terminal operator, and related customers, is considered. After unloading from vessels, inbound containers of the customers may stay in the container terminal yard (CTY) until the external trucks come to collect them. The inbound container dwell time $T$ (random variable) is the time interval between the container arrival time at the terminal and the time when the customer calls the inbound container. In the free-time contract system, if the container dwell time in the terminal yard is longer than $F_{0}$ days, the free-time-limit provided by the CTY, the carrier needs to pay the terminal operator $p_{0}$ per container per day. In the free-space contract system, if the occupied space in the container terminal yard exceeds $K$ (unit of $K$ is $\mathrm{TEU} *$ day), the carrier needs to pay the container terminal operator $p_{1}$ per container per day. We assume that there is an alternative storage place for the inbound containers $C$, a remote container storage yard (RCY) near the container terminal. The RCY provides storage space for the inbound containers and charges a storage fee $s_{0}$ per container per day: $s_{0}<p_{0}$ and $s_{0}<p_{1}$. However, the carrier needs to pay additional handling and transportation costs $c_{0}$ per container to move a container from the CTY to the RCY. 
The average number of arriving inbound containers at the terminal every day is $n$ TEU. It is widely accepted that the average number of inbound containers is independent of the storage price. For the simplicity, we assume that the average number of arriving containers at the container terminal every day is stable. Due to information asymmetry, the ocean carrier, CTY, and RCY do not know the exact inbound container dwell time; they only know the distribution $f(T)$ (probability density function). Following the analysis in the literature (K. H. Kim and K. Y. Kim [5]; Watanabe [10]), we assume that the inbound container dwell time follows exponential distribution $f(T)=\lambda e^{-\lambda T}$.

In the free-time contract system, facing the inbound container storage price scheme $\left(F_{0}, p_{0}\right)$, the ocean carrier decides the container storage fee $w$ and provides the price contract $\left(F_{0}, w\right)$ to the customer. It is assumed that $w>p_{0}$. In the free-space contract system, facing the inbound container storage price scheme $\left(K, p_{1}\right)$, the ocean carrier decides price contract $(F, s)$ to the customer. It is assumed that $s>p_{1}$.

Besides the container terminal yard, the customer has an alternative container storage place, that is, the RCY. Firstly, the ocean carrier and RCY will simultaneously provide their storage price schemes, $(F, s)$ (or $\left(F_{0}, w\right)$ for the free-time contract system) and $s_{0}$. After the storage price schemes are announced, the customer determines whether to transfer the inbound container from the CTY to the RCY after the free-time-limit. Figure 1 demonstrates the customer payment function. Here, we define $t_{s}=c_{0} /\left(s-s_{0}\right)\left(\right.$ or $t_{w}=c_{0} /\left(w-s_{0}\right)$ for the free-time contract system) as the indifference time. If the inbound container's dwell time is shorter than $F+t_{s}\left(\right.$ or $F_{0}+t_{w}$ for the free-time contract system), the customer will leave the container in the CTY till it is needed. If the container dwell time is longer than $F+t_{s}\left(\right.$ or $F_{0}+t_{w}$ ), then the customer will move the container to the RCY after the free-time-limit and then collect it when it is needed.

In the following two sections, we will study the ocean carrier's storage price schemes in the free-time contract system, $\left(F_{0}, w\right)$, and in the free-space contract system, $(F, s)$. In the free-time contract system, the free-time-limit value, $F_{0}$, is given by the container terminal. Hence, the ocean carrier only needs to determine the inbound container storage fee per day per TEU, $w$. In the free-space contract system, the two decisions of the ocean carrier are the free-time-limit $F$ and the inbound container storage fee $s$.

\section{Free-Time Contract System}

In the free-time contract system, the container terminal operator provides a free-time-limit $F_{0}$ and the inbound container extra storage fee rate $p_{0}$ to the ocean carrier. The ocean carrier offers the same free-time-limit $F_{0}$ to the customer but will add an additional storage fee. Namely, the ocean carrier's inbound container storage fee for the customer, $w$, is higher than $p_{0}$. It is assumed that the upper limit of the ocean carrier's charge is $\bar{w}$. Therefore, we have $p_{0} \leq w \leq \bar{w}$.

Based on the customer behavior analysis in Section 3, the containers, whose dwell time is less than $F_{0}+t_{w}$, will be stored

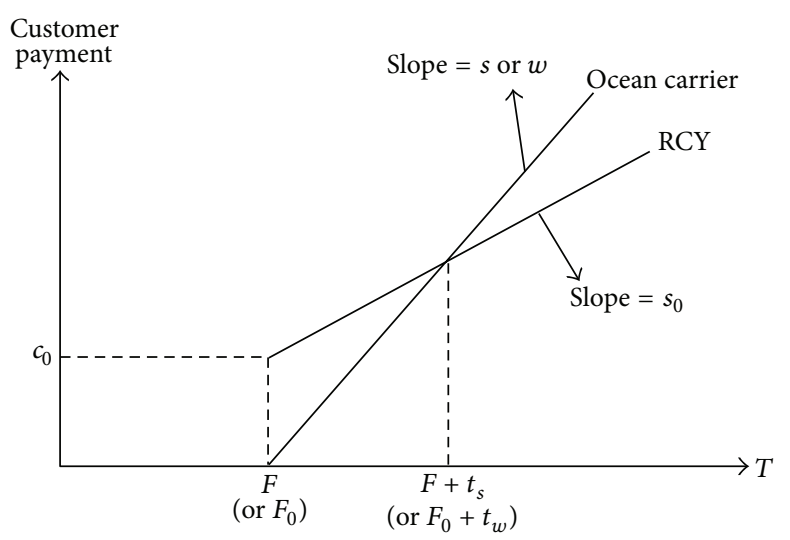

FIGURE 1: The payment function of the customer.

in the container terminal after the free-time-limit. Hence, the ocean carrier's profit is the following:

$$
\Pi(w)=n \int_{F_{0}}^{F_{0}+t_{w}}\left(w-p_{0}\right)\left(t-F_{0}\right) f(t) d t,
$$

where $\left(w-p_{0}\right)$ is the extra money the ocean carrier earns per TEU, besides paying the container terminal.

We can get the first and second derivatives of the ocean carrier's objective function as follows:

$$
\begin{aligned}
\frac{\partial \Pi}{\partial w}= & n \int_{F_{0}}^{F_{0}+t_{w}}\left(t-F_{0}\right) f(t) d t \\
& -n\left(w-p_{0}\right) \frac{c_{0}^{2}}{\left(w-s_{0}\right)^{3}} f\left(F_{0}+t_{w}\right), \\
\frac{\partial^{2} \Pi}{\partial w^{2}}= & -\frac{2 n c_{0}^{2}}{\left(w-s_{0}\right)^{3}} f\left(F_{0}+t_{w}\right)+\frac{3 n c_{0}^{2}\left(w-p_{0}\right)}{\left(w-s_{0}\right)^{4}} f\left(F_{0}+t_{w}\right) \\
& +\frac{n c_{0}^{3}\left(w-p_{0}\right)}{\left(w-s_{0}\right)^{5}} \frac{\partial f\left(F_{0}+t_{w}\right)}{\partial t_{w}} .
\end{aligned}
$$

Proposition 1. In the free-time contract system, the ocean carrier's objective function is concave if the following assumption is satisfied.

Assumption 2. $\bar{w} \leq 3 p_{0}-2 s_{0}$.

Proof. By (3), to prove the concavity of the ocean carrier's objective function, we need to show that

$$
\begin{aligned}
\frac{\partial^{2} \Pi}{\partial w^{2}}= & -\frac{2 n c_{0}^{2}}{\left(w-s_{0}\right)^{3}} f\left(F_{0}+t_{w}\right)+\frac{3 n c_{0}^{2}\left(w-p_{0}\right)}{\left(w-s_{0}\right)^{4}} f\left(F_{0}+t_{w}\right) \\
& +\frac{n c_{0}^{3}\left(w-p_{0}\right)}{\left(w-s_{0}\right)^{5}} \frac{\partial f\left(F_{0}+t_{w}\right)}{\partial t_{w}} \leq 0 .
\end{aligned}
$$


Since we have $f(T)=\lambda e^{-\lambda T}$, then

$$
\begin{aligned}
\frac{\partial^{2} \Pi}{\partial w^{2}}= & -\frac{2 n c_{0}^{2}}{\left(w-s_{0}\right)^{3}} \lambda e^{-\lambda\left(F_{0}+t_{w}\right)}+\frac{3 n c_{0}^{2}\left(w-p_{0}\right)}{\left(w-s_{0}\right)^{4}} \lambda e^{-\lambda\left(F_{0}+t_{w}\right)} \\
& -\frac{n c_{0}^{3}\left(w-p_{0}\right)}{\left(w-s_{0}\right)^{5}} \lambda^{2} e^{-\lambda\left(F_{0}+t_{w}\right)}
\end{aligned}
$$

We have $w>p_{0}$ and $w>s_{0}$; hence, the third part in the right side of the above expression is less than zero. If we have the assumption $\bar{w} \leq 3 p_{0}-2 s_{0}$, then we can get the first and second parts:

$$
-\frac{2 n c_{0}^{2}}{\left(w-s_{0}\right)^{3}} \lambda e^{-\lambda\left(F_{0}+t_{w}\right)}+\frac{3 n c_{0}^{2}\left(w-p_{0}\right)}{\left(w-s_{0}\right)^{4}} \lambda e^{-\lambda\left(F_{0}+t_{w}\right)} \leq 0 .
$$

Therefore, we have $\partial^{2} \Pi / \partial w^{2} \leq 0$.

Assumption 2 indicates that, given the RCY storage fee and the container terminal storage fee, the ocean carrier's storage price upper limit should not be too high. Otherwise, the container terminal could set pretty high storage price (even though there exists threat from the RCY), which is not fair to the customer.

Letting (2) equal zero, we can find the stationary point for the objective function. From Proposition 1, we know that if Assumption 2 is satisfied, then the objective function is concave in the feasible region $\left(p_{0}, \bar{w}\right)$ and there exists at most one stationary point in this region. If there is no stationary point in the feasible region, then the objective function either increases or decreases in $\left(p_{0}, \bar{w}\right)$. We summarize the solution steps as follows.

Step 1. Let (2) equal zero and use the line search method to find the stationary point. Go to Step 2.

Step 2. Check the $w$ value region $\left(p_{0}, \bar{w}\right)$; if there is one stationary point in this region, then let this point value be $w^{*}$, the optimal storage price, and stop. Otherwise, go to Step 3.

Step 3. If there is no stationary point in region $\left(p_{0}, \bar{w}\right)$ and the objective function is increasing in this region, then let $w^{*}=\bar{w}$ be the optimal storage price. On the other hand, if the objective function is decreasing in this region, then let $w^{*}=p_{0}$ be the optimal storage price. Stop.

\section{Free-Space Contract System}

In the free-space contract system, the container terminal operator provides an inbound container free-storage-space $K$ and the inbound container extra storage fee rate $p_{1}$ to the ocean carrier. If the total amount of space occupied by the inbound containers in the terminal exceeds $K$, then the ocean carrier needs to pay $p_{1}$ per TEU per day. Facing this price contract from the container terminal, the ocean carrier offers a free-time-limit $F$ and the inbound container extra storage fee rate $s$ to the customer. If the container dwell time in the terminal is longer than $F$, the customer needs to pay the ocean carrier $s$ per TEU per day. It is assumed that the ocean carrier charges more than the container terminal and the upper limit of the ocean carrier's charge is $\bar{s}$. Therefore, we have $p_{1} \leq s \leq$ $\bar{s}$.

The customer will leave their container in the container terminal after the free-time-limit if the dwell time is less than $F+t_{s}$. Therefore, the ocean carrier's profit is the following:

$$
\begin{aligned}
\Pi(F, s)= & n \int_{F}^{F+t_{s}} s(t-F) f(t) d t \\
& -p_{1}\left(\int_{0}^{F+t_{s}} n t f(t) d t-K\right)^{+},
\end{aligned}
$$

where the first part is the ocean carrier's income from the containers whose dwell time is less than $F+t_{s}$. In the second part, $\int_{0}^{F+t_{s}} n t f(t) d t$ denotes the expected space occupied by containers in the container terminal yard. Hence, the second part is the ocean carrier's payment to the container terminal for the containers whose space exceeds the free-space value, $K$.

Proposition 3. In the free-space contract system, the ocean carrier's optimal free-time-limit setting is zero; namely, $F^{*}=0$.

Proof. To prove $F^{*}=0$, we only need to show that, for any given $s$, the optimal value of $F$ is 0 . We divide (7) into two parts to analyze the monotonicity. Firstly, for the first part, we take derivative with $F$ and have

$$
\begin{aligned}
& \frac{\partial\left(n \int_{F}^{F+t_{s}} s(t-F) f(t) d t\right)}{\partial F} \\
& \quad=-n s \int_{F}^{F+t_{s}} f(t) d t+n s t_{s} f\left(F+t_{s}\right) .
\end{aligned}
$$

Since the container dwell time follows exponential distribution, we can draw out its distribution as in Figure 2. From the figure, we can easily get $\int_{F}^{F+t_{s}} f(t) d t \geq t_{s} f\left(F+t_{s}\right)$. Therefore, we have $-n s \int_{F}^{F+t_{s}} f(t) d t+n s t_{s} f\left(F+t_{s}\right) \leq 0$. Namely, the first part of the objective function decreases with $F$. Hence, for the first part, the optimal value of $F$ is 0 .

We now investigate the second part of (7), $-p_{1}\left(\int_{0}^{F+t_{s}} n t f(t) d t-K\right)^{+}$. In the second part, $\int_{0}^{F+t_{s}} n t f(t) d t$ increases with $F$. Therefore, the second part decreases with $F$. Hence, for the second part, the optimal value of $F$ is also 0 .

Summarizing the analysis of the first and second parts, we have $F^{*}=0$.

Although the optimal value of the free-time-limit is zero, it is often set to a positive value by the ocean carrier in practice. Therefore, in the remainder of this section, we take it as given and focus on the study of the optimal storage price $s$.

Followed by Proposition 3, we can rewrite (7) as

$$
\Pi(s)=n \int_{0}^{t_{s}} s t f(t) d t-p_{1}\left(\int_{0}^{t_{s}} n t f(t) d t-K\right)^{+} .
$$




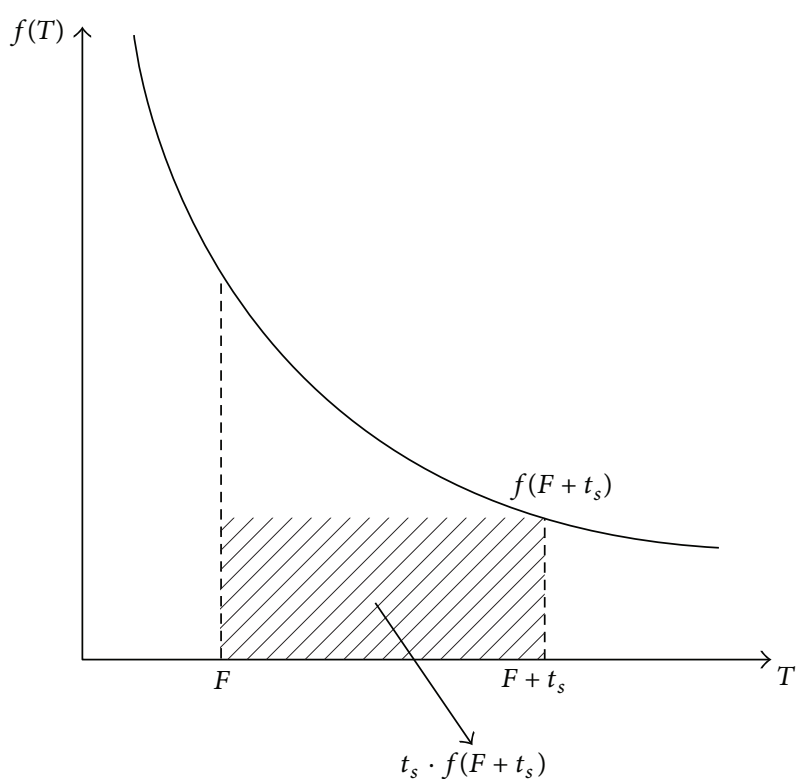

FIgURE 2: The container dwell time distribution.

Let $\int_{0}^{t_{s}} n t f(t) d t-K=0$; then, we solve a corresponding $s$ value and let this value be $s_{H}$. Since $t_{s}=c_{0} /\left(s-s_{0}\right)$, it is easy to find that $\int_{0}^{t_{s}} n t f(t) d t$ decreases with $s$. By this monotonic property, we can rewrite (9) to two segments and get

$$
\Pi(s)=\left\{\begin{array}{lr}
\Pi_{1}(s)=n \int_{0}^{t_{s}} s t f(t) d t, & \text { if } s \geq s_{H}, \\
\Pi_{2}(s)=n \int_{0}^{t_{s}}\left(s-p_{1}\right) t f(t) d t+p_{1} K & \text { otherwise. }
\end{array}\right.
$$

We now derive the conditions under which $\Pi_{1}(s)$ and $\Pi_{2}(s)$ are concave. The first and second derivatives of $\Pi_{1}(s)$ and $\Pi_{2}(s)$ can be obtained as follows:

$$
\begin{aligned}
& \frac{\partial \Pi_{1}(s)}{\partial s}= n \int_{0}^{t_{s}} t f(t) d t-\frac{c_{0} n s t_{s}}{\left(s-s_{0}\right)^{2}} f\left(t_{s}\right), \\
& \frac{\partial^{2} \Pi_{1}(s)}{\partial s^{2}}=-\frac{2 n c_{0}^{2}}{\left(s-s_{0}\right)^{3}} f\left(t_{s}\right)+\frac{3 n s c_{0}^{2}}{\left(s-s_{0}\right)^{4}} f\left(t_{s}\right) \\
&+\frac{n s c_{0}^{3}}{\left(s-s_{0}\right)^{5}} \frac{\partial f\left(t_{s}\right)}{\partial t_{s}}, \\
& \frac{\partial \Pi_{2}(s)}{\partial s}=n \int_{0}^{t_{s}} t f(t) d t-\frac{c_{0} n\left(s-p_{1}\right) t_{s}}{\left(s-s_{0}\right)^{2}} f\left(t_{s}\right), \\
& \frac{\partial^{2} \Pi_{2}(s)}{\partial s^{2}}=-\frac{2 n c_{0}^{2}}{\left(s-s_{0}\right)^{3}} f\left(t_{s}\right)+\frac{3 n\left(s-p_{1}\right) c_{0}^{2}}{\left(s-s_{0}\right)^{4}} f\left(t_{s}\right) \\
&+\frac{n\left(s-p_{1}\right) c_{0}^{3} \frac{\partial f\left(t_{s}\right)}{\left(s-s_{0}\right)^{5}} .}{\partial t_{s}}
\end{aligned}
$$

Proposition 4. In the free-space contract system, $\Pi_{1}(s)$ is concave if the following assumption is satisfied.

Assumption 5. $\bar{s} \leq\left(\lambda c_{0}-s_{0}+\sqrt{\left(s_{0}-\lambda c_{0}\right)^{2}+8 s_{0}^{2}}\right) / 2$.

Proof. To prove Proposition 4, we need to show that if $\bar{s} \leq$ $\left(\lambda c_{0}-s_{0}+\sqrt{\left(s_{0}-\lambda c_{0}\right)^{2}+8 s_{0}^{2}}\right) / 2$, then

$$
\begin{aligned}
\frac{\partial^{2} \Pi_{1}(s)}{\partial s^{2}}= & -\frac{2 n c_{0}^{2}}{\left(s-s_{0}\right)^{3}} f\left(t_{s}\right)+\frac{3 n s c_{0}^{2}}{\left(s-s_{0}\right)^{4}} f\left(t_{s}\right) \\
& +\frac{n s c_{0}^{3}}{\left(s-s_{0}\right)^{5}} \frac{\partial f\left(t_{s}\right)}{\partial t_{s}} \leq 0 .
\end{aligned}
$$

Since we have $f(T)=\lambda e^{-\lambda T}$, hence

$$
\begin{aligned}
\frac{\partial^{2} \Pi_{1}(s)}{\partial s^{2}}= & -\frac{2 n c_{0}^{2}}{\left(s-s_{0}\right)^{3}} \lambda e^{-\lambda t_{s}}+\frac{3 n s c_{0}^{2}}{\left(s-s_{0}\right)^{4}} \lambda e^{-\lambda t_{s}} \\
& -\frac{n s c_{0}^{3}}{\left(s-s_{0}\right)^{5}} \lambda^{2} e^{-\lambda t_{s}} \\
= & \frac{n \lambda e^{-\lambda t_{s}} c_{0}^{2}}{\left(s-s_{0}\right)^{5}}\left(s^{2}+s_{0} s-\lambda c_{0} s-2 s_{0}^{2}\right) .
\end{aligned}
$$

If $\bar{s} \leq\left(\lambda c_{0}-s_{0}+\sqrt{\left(s_{0}-\lambda c_{0}\right)^{2}+8 s_{0}^{2}}\right) / 2$, then we have $\left(s^{2}+s_{0} s-\right.$ $\left.\lambda c_{0} s-2 s_{0}^{2}\right) \leq 0$. Therefore, if Assumption 5 is satisfied, then $\partial^{2} \Pi_{1}(s) / \partial s^{2} \leq 0$.

Proposition 6. In the free-space contract system, $\Pi_{2}(s)$ is concave if Assumption 5 and the following assumption are satisfied.

Assumption 7. $p_{1} \geq\left(\lambda c_{0}+3 p_{1}-s_{0}-\right.$ $\sqrt{\left.\left(s_{0}-\lambda c_{0}-3 p_{1}\right)^{2}+8 s_{0}^{2}-12 p_{1} s_{0}-4 \lambda c_{0} p_{1}\right)} / 2$.

Proof. To prove Proposition 6, we need to show that if Assumption 5 is satisfied and $p_{1} \geq\left(\lambda c_{0}+3 p_{1}-s_{0}-\right.$ $\sqrt{\left.\left(s_{0}-\lambda c_{0}-3 p_{1}\right)^{2}+8 s_{0}^{2}-12 p_{1} s_{0}-4 \lambda c_{0} p_{1}\right)} / 2$, then

$$
\begin{aligned}
\frac{\partial^{2} \Pi_{2}(s)}{\partial s^{2}}= & -\frac{2 n c_{0}^{2}}{\left(s-s_{0}\right)^{3}} f\left(t_{s}\right)+\frac{3 n\left(s-p_{1}\right) c_{0}^{2}}{\left(s-s_{0}\right)^{4}} f\left(t_{s}\right) \\
& +\frac{n\left(s-p_{1}\right) c_{0}^{3}}{\left(s-s_{0}\right)^{5}} \frac{\partial f\left(t_{s}\right)}{\partial t_{s}} \leq 0 .
\end{aligned}
$$


Since we have $f(T)=\lambda e^{-\lambda T}$, hence

$$
\begin{aligned}
\frac{\partial^{2} \Pi_{2}(s)}{\partial s^{2}}= & -\frac{2 n c_{0}^{2}}{\left(s-s_{0}\right)^{3}} \lambda e^{-\lambda t_{s}}+\frac{3 n\left(s-p_{1}\right) c_{0}^{2}}{\left(s-s_{0}\right)^{4}} \lambda e^{-\lambda t_{s}} \\
& -\frac{n\left(s-p_{1}\right) c_{0}^{3}}{\left(s-s_{0}\right)^{5}} \lambda^{2} e^{-\lambda t_{s}} \\
= & \frac{n \lambda e^{-\lambda t_{s}} c_{0}^{2}}{\left(s-s_{0}\right)^{5}}\left[s^{2}+\left(s_{0}-\lambda c_{0}-3 p_{1}\right) s-2 s_{0}^{2}\right. \\
& \left.+3 p_{1} s_{0}+\lambda c_{0} p_{1}\right] .
\end{aligned}
$$

We only need to show that $s^{2}+\left(s_{0}-\lambda c_{0}-3 p_{1}\right) s-2 s_{0}^{2}+3 p_{1} s_{0}+$ $\lambda c_{0} p_{1} \leq 0$. From the property of the quadratic formula, we know that if the following inequations are satisfied,

$$
\begin{aligned}
& \left(\lambda c_{0}+3 p_{1}-s_{0}\right. \\
& \left.-\sqrt{\left(s_{0}-\lambda c_{0}-3 p_{1}\right)^{2}+8 s_{0}^{2}-12 p_{1} s_{0}-4 \lambda c_{0} p_{1}}\right) \times \frac{1}{2} \\
& \leq s \leq\left(\lambda c_{0}+3 p_{1}-s_{0}\right. \\
& \left.\quad+\sqrt{\left(s_{0}-\lambda c_{0}-3 p_{1}\right)^{2}+8 s_{0}^{2}-12 p_{1} s_{0}-4 \lambda c_{0} p_{1}}\right) \\
& \quad \times \frac{1}{2}=A\left(p_{1}\right),
\end{aligned}
$$

then $s^{2}+\left(s_{0}-\lambda c_{0}-3 p_{1}\right) s-2 s_{0}^{2}+3 p_{1} s_{0}+\lambda c_{0} p_{1} \leq 0$.

We now prove the right inequation of (19). It is easy to find that $\partial A\left(p_{1}\right) / \partial p_{1}>0$. Since we have $p_{1}>0$, we get $A\left(p_{1}>\right.$ $0)>A\left(p_{1}=0\right)=\left(\lambda c_{0}-s_{0}+\sqrt{\left(s_{0}-\lambda c_{0}\right)^{2}+8 s_{0}^{2}}\right) / 2$. Because $s \leq \bar{s}$, if Assumption 5 is satisfied, then the right inequation of (19) is satisfied.

Because $s \geq p_{1}$, if Assumption 7 is satisfied, then the left inequation of (19) is also satisfied.

Assumption 5 reveals that, given other parameters, the ocean carrier should not set the storage price too high (namely, the storage price upper limit is constrained). Otherwise, the unreasonable high storage price is unfair to the customer. Assumption 7 indicates that the ocean carrier could not choose too low storage price (namely, the storage price lower limit is also constrained). Otherwise, the inbound containers stay too long in the container terminal and occupy a large amount of space, which hurts both the container terminal (in space control) and the carrier (in storage payment).

From Propositions 4 and 6, we know that if Assumptions 5 and 7 are satisfied, then there exists at most one stationary point of the objective function in the feasible region $\left(p_{1}, \bar{s}\right)$ for both $\Pi_{1}(s)$ and $\Pi_{2}(s)$. If the feasible region contains no stationary point, then the objective function either increases or decreases in this region. We summarize the solution steps as follows.
Step 1. Solve $\int_{0}^{t_{s}} n t f(t) d t-K=0$ and get a corresponding $s$ value. Let this value be $s_{H}$.

Step 2. If $s_{H} \geq \bar{s}$, we have the objective function $\Pi(s)=\Pi_{2}(s)$, let (13) be equal to zero, and use the line search method to find the stationary point, and then we go to Step 3 . If $s_{H} \leq p_{1}$, we have the objective function $\Pi(s)=\Pi_{1}(s)$, let (11) be equal to zero to find the stationary point, and then go to Step 3. If $p_{1}<$ $s_{H}<\bar{s}$, then go to Step 4 .

Step 3. Check the region $\left(p_{1}, \bar{s}\right)$.

(1) If there is one stationary point in this region, then let this point value be $s^{*}$.

(2) If there is no stationary point in region $\left(p_{1}, \bar{s}\right)$ and the objective function is increasing in this region, then let $s^{*}=\bar{s}$ be the optimal storage price.

(3) If there is no stationary point in region $\left(p_{1}, \bar{s}\right)$ and the objective function is decreasing in this region, then let $s^{*}=p_{1}$ be the optimal storage price.

Stop.

Step 4. Separate the region $\left(p_{1}, \bar{s}\right)$ into two parts: $\left(p_{1}, s_{H}\right)$ and $\left(s_{H}, \bar{s}\right)$.

Step 5. For part $\left(s_{H}, \bar{s}\right)$, we have the objective function $\Pi(s)=$ $\Pi_{1}(s)$. Let (11) be equal to zero and find the stationary point.

(1) If the stationary point is in region $\left(s_{H}, \bar{s}\right)$, let the $s_{1}^{*}$ be this stationary point.

(2) If there is no stationary point in region $\left(s_{H}, \bar{s}\right)$ and $\Pi_{1}(s)$ is increasing in this region, then let $s_{1}^{*}=\bar{s}$ be the optimal storage price.

(3) If there is no stationary point in region $\left(s_{H}, \bar{s}\right)$ and $\Pi_{1}(s)$ is decreasing in this region, then let $s_{1}^{*}=s_{H}$ be the optimal storage price.

Step 6. For part $\left(p_{1}, s_{H}\right)$, we have the objective function $\Pi(s)=\Pi_{2}(s)$. Let (13) be equal to zero and find the stationary point.

(1) If the stationary point is in region $\left(p_{1}, s_{H}\right)$, let the $s_{2}^{*}$ be this stationary point.

(2) If there is no stationary point in region $\left(p_{1}, s_{H}\right)$ and $\Pi_{2}(s)$ is increasing in this region, then let $s_{2}^{*}=s_{H}$ be the optimal storage price.

(3) If there is no stationary point in region $\left(p_{1}, s_{H}\right)$ and $\Pi_{2}(s)$ is decreasing in this region, then let $s_{2}^{*}=p_{1}$ be the optimal storage price.

Step 7. Let $s^{*}=\operatorname{argmax}\left\{\Pi_{1}\left(s_{1}^{*}\right), \Pi_{2}\left(s_{2}^{*}\right)\right\}$. Stop.

\section{Numerical Experiments}

In order to verify the effectiveness of the proposed models and their solution methods, several numerical experiments are conducted. The analysis in this section lies in the following threefold: (1) the effect of the container terminal's contract 
TABLE 1: Parameters setting for numerical experiments.

\begin{tabular}{lc}
\hline Parameters & Applied value \\
\hline $\begin{array}{l}\text { Average number of arriving inbound containers } \\
\text { every day, } n\end{array}$ & 1000 TEU \\
$\begin{array}{l}\text { Inbound container dwell time distribution } \\
\text { parameter, } \lambda\end{array}$ & 0.6 \\
$\begin{array}{l}\text { The container transportation cost from the CTY } \\
\text { to the RCY, } c_{0}\end{array}$ & $\$ 100$ \\
$\begin{array}{l}\text { The container storage fee in the RCY, } s_{0} \\
\text { The carrier's storage fee uplimit in free-time }\end{array}$ & $\$ 45$ \\
system, $\bar{w}$ & $\$ 70$ \\
$\begin{array}{l}\text { The carrier's storage fee uplimit in free-space } \\
\text { system, } \bar{s}\end{array}$ & $\$ 70$
\end{tabular}

settings on the three parties (e.g., the carrier, the container terminal, and the customer) is studied; (2) the sensitivity analysis with respect to the parameter changes (e.g., the container dwell time distribution, the container transportation cost from the CTY to the RCY, and the storage fee in the RCY) is conducted; (3) the two contract systems are compared under the same conditions so as to investigate the carrier's profit, the container terminal's income, and the customer's cost.

Before presenting the numerical results, we first provide the parameter and condition settings in the following subsection.

6.1. Preliminary Setting. In order to guarantee that the two contract systems are compared under the same condition, we set the container terminal's two kinds of contract parameters as follows.

(1) The storage fees charged by the container terminal to the carrier under the two systems are set the same: $p_{0}=p_{1}$. Similarly, the carrier's storage fee uplimits are set the same: $\bar{w}=\bar{s}$.

(2) The free-storage-space $K$ offered by the container terminal in the free-space contract system equals the equivalent space accumulated in the free-time-limit $F_{0}$ in the free-time system. Namely, we have $K=$ $\int_{0}^{F_{0}} n t f(t) d t$.

For the container terminal parameter effect analysis and the comparison of the two contract systems, we set the common parameters as in Table 1 . All parameters are set such that Assumptions 2, 5 and 7 are satisfied. In each instance, we change one parameter and keep other parameters unchanged. In order to better demonstrate the effect of different parameters on carrier's price, customer's cost, carrier's profit, container terminal operator's income, and the average storage space in the container terminal at the same time, we use tables rather than figures to show the results.

For the sensitivity analysis, we change the value of $c_{0}, s_{0}$, and $\lambda$, so as to study the system outcomes. Parameters settings are described later in Tables 3 and 4 .
6.2. The Effect of the Container Terminal Contract Parameters. In this subsection, the effect of the container terminal's storage contract parameter change on the system outcomes under the two contract systems is first investigated. We keep other parameters unchanged and only adjust the free-timelimit $F_{0}$ (or the free-storage-space $K$ ) and the container terminal storage fee $p_{0}$ ( or $p_{1}$ ). The results are summarized in Table 2. We use "FT" and "FS" to denote the free-time and free-space contract systems in the table. By these numerical analyses, we intend to figure out the container terminal's reasonable decisions on the free-time-limit, the free-storagespace value, and the storage fee.

From the numerical results in Table 2, we summarize the insights from three angles: the container terminal, the carrier, and the customers.

\subsubsection{Container Terminal (CT)}

(1) In the free-time contract system, with the rise of the free-time-limit $F_{0}$, the average storage space in the container terminal occupied by the inbound containers will increase. Moreover, the longer free-timelimit leads to lower income to the container terminal. Therefore, it is suggested that the container terminal should not set too long free storage time in the free-time contract system. Practically, the container terminal often provides a nonzero free-time-limit directly to the carrier (or indirectly to the customer). Although this value could be different among different carriers, depending on the relationship between the carrier and the terminal operator, the container terminal operator should not set the value too high according to our analysis.

(2) In the free-space contract system, when the freestorage-space value $K$ is relatively small, the container terminal's income (and its percentage of the customer's total payment) increases with the terminal's storage fee $\left(p_{1}\right)$. When the free space value $K$ is large, the carrier takes away all of the customer's payment and the container terminal gets no income. In addition, with the increase of $K$, the average storage space in the terminal occupied by the containers increases. Hence, the container terminal should not set too large $K$ value, for the sake of traffic control and income enhancement.

(3) The container terminal and the carrier share the customer's storage payment (namely, each one takes a proportion). In the free-time contract system, the container terminal's income (and its percentage) increases with the terminal's storage fee $\left(p_{0}\right)$. This result is intuitive, because the higher the storage charge, the higher the container terminal's income.

\subsubsection{Carrier and Customer}

(1) In the free-time contract system, the carrier's profit and the customer's cost decrease with the free-timelimit $\left(F_{0}\right)$ set by the container terminal, which is quite 
TABLE 2: Numerical results under different container terminal storage contract parameter settings.

\begin{tabular}{|c|c|c|c|c|c|c|c|c|c|c|c|c|}
\hline \multirow{2}{*}{\multicolumn{2}{|c|}{$\begin{array}{l}\text { CT storage } \\
\text { contract } \\
\text { parameters } \\
F_{0} p_{0}\left(p_{1}\right) \\
\end{array}$}} & \multirow[b]{2}{*}{ K } & \multicolumn{2}{|c|}{$\begin{array}{l}\text { Carrier } \\
\text { price }(\$)\end{array}$} & \multicolumn{2}{|c|}{$\begin{array}{l}\text { Customer cost }(\$) \\
\text { (carrier revenue) }\end{array}$} & \multicolumn{2}{|c|}{ Carrier profit (\$) } & \multicolumn{2}{|c|}{ CT income (\$) } & \multicolumn{2}{|c|}{$\begin{array}{l}\text { Average storage } \\
\text { space (TEU } * \text { day) }\end{array}$} \\
\hline & & & FT & FS & FT & FS & FT & FS & FT & FS & FT & FS \\
\hline & 55 & 895.3 & 70 & 70 & 13346.7 & 80681.8 & $2860.0(21.4 \%)$ & $66528.9(82.5 \%)$ & $10486.7(78.6 \%)$ & $14152.9(17.5 \%)$ & 1537.1 & $1152.6>K$ \\
\hline & 60 & 895.3 & 70 & 70 & 13346.7 & 80681.8 & $1906.7(14.3 \%)$ & $65242.2(80.9 \%)$ & $11440.0(85.7 \%)$ & $15439.6(19.1 \%)$ & 1537.1 & $1152.6>K$ \\
\hline 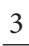 & 65 & 895.3 & 70 & 70 & 13346.7 & 80681.8 & $953.3(7.1 \%)$ & $63955.6(79.3 \%)$ & $12393.4(92.9 \%)$ & $16726.2(20.7 \%)$ & 1537.1 & $1152.6>K$ \\
\hline 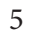 & 55 & 1335.5 & 70 & 65 & 4019.9 & 86807.3 & $861.4(21.4 \%)$ & $86807.3(100 \%)$ & $3158.5(78.6 \%)$ & $0(0 \%)$ & 1618.5 & $1335.5=K$ \\
\hline D & 60 & 1335.5 & 70 & 65 & 4019.9 & 86807.3 & $574.3(14.3 \%)$ & $86807.3(100 \%)$ & $3445.6(85.7 \%)$ & $0(0 \%)$ & 1618.5 & $1335.5=K$ \\
\hline 5 & 65 & 1335.5 & 70 & 65 & 4019.9 & 86807.3 & $287.1(7.1 \%)$ & $86807.3(100 \%)$ & $3732.8(92.9 \%)$ & $0(0 \%)$ & 1618.5 & $1335.5=K$ \\
\hline 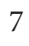 & 55 & 1537.1 & 70 & 59.3 & 1210.8 & 91130.6 & $259.5(21.4 \%)$ & $91130.6(100 \%)$ & $951.3(78.6 \%)$ & $0(0 \%)$ & 1649.5 & $1537.1=K$ \\
\hline 7 & 60 & 1537.1 & 70 & 60 & 1210.8 & 90842.0 & $173.0(14.3 \%)$ & $90842.0(100 \%)$ & $1037.8(85.7 \%)$ & $0(0 \%)$ & 1649.5 & $1514.0<K$ \\
\hline 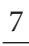 & 65 & 1537.1 & 70 & 65 & 1210.8 & 86807.3 & $86.5(7.1 \%)$ & $86807.3(100 \%)$ & $1124.3(92.9 \%)$ & $0(0 \%)$ & 1649.5 & $1335.5<K$ \\
\hline
\end{tabular}

${ }^{1} \mathrm{FT}=$ free-time contract system; FS = free-space contract system.

${ }^{2}$ The percentages in the table denote the proportions of the carrier's profits or container terminal's incomes to the customer payment costs.

TABLE 3: Sensitivity analysis of free-time system under different $c_{0}, s_{0}$, and $\lambda$.

\begin{tabular}{cccccccc}
\hline \multicolumn{2}{c}{ Parameters } & Carrier price (\$) & $\begin{array}{l}\text { Customer cost }(\$) \\
\text { (carrier revenue) }\end{array}$ & Carrier profit (\$) & CT income (\$) & $\begin{array}{l}\text { Average storage space } \\
(\text { TEU } * \text { day) }\end{array}$ \\
\hline 25 & 45 & 0.2 & 66.7 & 4164.2 & $732(17.6 \%)$ & $3432.2(82.4 \%)$ & 1010.3 \\
35 & 45 & 0.2 & 67.5 & 7302.8 & $1352.7(18.5 \%)$ & $5950.1(81.5 \%)$ & 1157.8 \\
45 & 45 & 0.2 & 68.3 & 10855.0 & $2113.4(19.5 \%)$ & $8741.6(80.5 \%)$ & 1296.0 \\
\hline 45 & 35 & 0.2 & 70 & 5360.0 & $1148.6(21.4 \%)$ & $4211.4(78.6 \%)$ & 1059.4 \\
45 & 40 & 0.2 & 70 & 7094.9 & $1520.3(21.4 \%)$ & $5574.6(78.6 \%)$ & 1137.6 \\
45 & 45 & 0.2 & 68.3 & 10855.0 & $2113.4(19.5 \%)$ & $8741.6(80.5 \%)$ & 1296.0 \\
\hline 25 & 45 & 0.2 & 66.7 & 4164.2 & $732.0(17.6 \%)$ & $3432.2(82.4 \%)$ & 1010.3 \\
25 & 45 & 0.3 & 67.7 & 4035.3 & $756.0(18.7 \%)$ & $3279.3(81.3 \%)$ & 1161.5 \\
25 & 45 & 0.4 & 68.7 & 3500.5 & $696.6(19.9 \%)$ & $2803.9(80.1 \%)$ & 1207.1 \\
\hline
\end{tabular}

${ }^{1}$ The parameters are set as follows: $F_{0}=7$ days; $p_{0}=\$ 55 ; \bar{w}=\$ 70$.

${ }^{2}$ The percentages in the table denote the proportions of the carrier's profits or container terminal's incomes to the customer payment costs.

intuitive. But in the free-space contract system, the carrier's profit and the customer's cost increase (or at least do not decrease) with the free-storage-space $(K)$ provided by the terminal. This is because the increase of the free-space value makes the carrier have more freely controllable space, storing more containers freely in the terminal but still charging the customers.

(2) The carrier earns less with the increase of the storage fee $\left(p_{0}\right.$ or $\left.p_{1}\right)$ of the container terminal, which is intuitive. The customer pays less (or at least not more) if the storage fee $\left(p_{0}\right.$ or $\left.p_{1}\right)$ set by the container terminal increases. The reason lies in the fact that the increase of the container terminal storage fee makes the carrier raise his price, which further drives the containers to the RCY. Therefore, the customer pays less by the container transfer.

6.3. Sensitivity Analysis. We now analyze how the change of the parameters (besides the container terminal's contract parameter) affects the system outcomes. The results are shown in Tables 3 and 4. Notations "FT" and "FS" are utilized to denote the free-time and free-space contract systems in the tables. The parameters are set as described in the footnotes of the tables. Assumptions 2, 5 and 7 are satisfied.

We summarize the results as follows.

6.3.1. Effect of $c_{0}$. In both the free-time and free-space contract systems, with the increase of the container transportation cost from the CTY to the RCY, $c_{0}$, the containers are more likely to be stored in the CTY. Therefore, the carrier has motivation to raise the storage price. The carrier and the terminal gain more incomes. The customer suffers both high transportation cost and high total storage payment.

6.3.2. Effect of $s_{0}$. In both the free-time and free-space contract systems, if the storage fee in the RCY rises, then customers are willing to store the containers in the CTY. Therefore, the profits of the carrier and the terminal increase.

6.3.3. Effect of $\lambda$. If there are more inbound containers to be collected early by the customer (namely, $\lambda$ increases), 
TABLE 4: Sensitivity analysis of free-space system under different $c_{0}, s_{0}$, and $\lambda$.

\begin{tabular}{cccccccc}
\hline \multicolumn{2}{c}{ Parameters } & Carrier price $(\$)$ & $\begin{array}{c}\text { Customer cost }(\$) \\
\text { (carrier revenue) }\end{array}$ & Carrier profit (\$) & CT income (\$) & $\begin{array}{c}\text { Average storage space } \\
(\text { TEU } * \text { day) }\end{array}$ \\
\hline 100 & 45 & 0.6 & 59.3 & 91130.6 & $91130.6(100 \%)$ & $0(0 \%)$ & $1537.1=K$ \\
110 & 45 & 0.6 & 60.7 & 93326.5 & $93326.5(100 \%)$ & $0(0 \%)$ & $1537.1=K$ \\
120 & 45 & 0.6 & 62.1 & 95522.4 & $95522.4(100 \%)$ & $0(0 \%)$ & $1537.1=K$ \\
\hline 100 & 45 & 0.6 & 59.3 & 91130.6 & $91130.6(100 \%)$ & $0(0 \%)$ & $1537.1=K$ \\
100 & 47 & 0.6 & 61.3 & 94204.9 & $94204.9(100 \%)$ & $0(0 \%)$ & $1537.1=K$ \\
100 & 50 & 0.6 & 64.3 & 98816.3 & $98816.3(100 \%)$ & $0(0 \%)$ & $1537.1=K$ \\
\hline 100 & 45 & 0.6 & 59.3 & 91130.6 & $91130.6(100 \%)$ & $0(0 \%)$ & $1537.1=K$ \\
100 & 45 & 0.7 & 61.1 & 81247.8 & $81247.8(100 \%)$ & $0(0 \%)$ & $1329.2<K$ \\
100 & 45 & 0.8 & 64.4 & 73846.9 & $73846.9(100 \%)$ & $0(0 \%)$ & $1145.9<K$ \\
\hline 100 & 45 & 0.6 & 70 & 80681.8 & $66528.9(82.5 \%)$ & $14152.9(17.5 \%)$ & $1152.6>K$ \\
110 & 45 & 0.6 & 70 & 86361.8 & $67746.0(78.4 \%)$ & $18615.8(21.6 \%)$ & $1233.7>K$ \\
120 & 45 & 0.6 & 70 & 91256.2 & $68794.8(75.4 \%)$ & $22461.4(24.6 \%)$ & $1303.7>K$ \\
\hline 100 & 45 & 0.6 & 70 & 80681.8 & $66528.9(82.5 \%)$ & $14152.9(17.5 \%)$ & $1152.6>K$ \\
100 & 47 & 0.6 & 70 & 85667.1 & $67597.2(78.9 \%)$ & $18069.9(21.1 \%)$ & $1223.8>K$ \\
100 & 50 & 0.6 & 70 & 93484.8 & $69272.4(74.1 \%)$ & $24212.4(25.9 \%)$ & $1335.5>K$ \\
\hline 100 & 45 & 0.6 & 70 & 80681.8 & $66528.9(82.5 \%)$ & $14152.9(17.5 \%)$ & $1152.6>K$ \\
100 & 45 & 0.7 & 70 & 76892.1 & $65221.4(84.8 \%)$ & $11670.7(15.2 \%)$ & $1098.5>K$ \\
100 & 45 & 0.8 & 70 & 72519.8 & $63084.6(87.0 \%)$ & $9435.2(13.0 \%)$ & $1036.0>K$ \\
\hline
\end{tabular}

${ }^{1}$ The parameters are set as follows: $p_{1}=\$ 55 ; \bar{s}=\$ 70$. In the first 9 instances, $K=1537.1$ TEU $*$ day, and in the second 9 instances, $K=895.3$ TEU $*$ day.

${ }^{2}$ The percentages in the table denote the proportions of the carrier's profits or container terminal's incomes to the customer payment costs.

then the customer totally pays less in both the free-time and free-space contract systems. This is because the earlier the containers are collected, the shorter the storage time is, which makes the customer pay less. In the free-space contract system, if the containers are to be collected earlier ( $\lambda$ increases), the carrier's profit percentage to the customer's payment increases (or at lease does not decrease). It is due to the reason that, facing a given free space from the container terminal, the earlier the containers are collected, the more free space to utilize the carrier has. Therefore, the carrier's profit percentage increases.

6.4. Comparison of the Two Contract Systems. The free-time contract system is widely used in the ocean transportation network. However, the free-space contract system is newly developed. Currently, there is no related research concerning the comparison of the two systems. After analyzing the two models in the previous sections, we now use the numerical experiments results (refer to Table 2) to quantitatively compare these two contract systems. We analyze the system outcomes from three aspects: the carrier, the customer, and the container terminal. The percentages in Table 2 denote the proportions of the carrier's profits or the terminal's incomes to the customer's payments.

\subsubsection{Carrier}

(1) The carrier makes more profit in the free-space contract system than in the free-time one. In the freetime system, the carrier only adds a small extra fee based on the price provided by the container terminal. However, in the free-space system, the carrier treats the free-storage-space provided by the container terminal as a pooling storage system. Although the dwell times of different containers vary from short to long, as long as the total storage space in the terminal is below the free-storage-space value $K$, the carrier pays nothing to the terminal. Therefore, the free-space contract system alleviates the container dwell time varying risk for the carrier and brings him a high profit.

(2) In the free-time contract system, the carrier receives a smaller proportion of the customer's payment than the container terminal, while, in the free-space contract system, the carrier takes larger proportion of the customer's payment than the terminal. In some cases, the carrier even sets suitable storage price such that the storage space occupied by the containers in the terminal is no more than $K$. Hence, the carrier earns $100 \%$ of the customer's storage payment.

Based on the analysis above, the carrier prefers the freespace contract system to the free-time one, because it brings him higher profit.

\subsubsection{Container Terminal (CT)}

(1) If the free-storage-space $K$ is small, then the container terminal earns more in the free-space contract system than in the free-time system, while if $K$ is relatively 
large, in the free-space contract system, the container terminal's income is zero. This is because, facing the large free-storage-space value, the carrier will set suitable storage price such that the container space in the terminal is less than or equal to $K$.

(2) Although the container terminal has the risk of no income from the container storage in the free-space contract system, this contract system can help to reduce the space occupied by the inbound containers in the CT. Therefore, for a busy container terminal, the free-space contract system is preferred by the terminal operator. After all, the container storage income is not the terminal's main focus. The terminal operators are eager to control the traffic congestion because the terminal yard crane operation cost quadratically increases with the number of containers in the yard [11]. Moreover, the terminal yard efficiency helps to increase the waterside quay crane operation speed.

(3) If the terminal operator has choice, he would rather not use either of the two contract systems but would directly provide the free-time contract to the customer because, in the free-time contract system and the free-space contract system, the carrier takes away all or part of the customer's payment, while if the direct free-time contract is signed between the container terminal and the customer, the terminal operator seizes $100 \%$ of the customer's storage payment.

From the discussion above, we know that, in the unaligned relationship ocean transportation network, the free-space contract system is a "win-win" strategy for both the carrier and the busy container terminal.

6.4.3. Customer. The customers pay more storage fee in the free-space contract system than in the free-time contract system. This is due to the fact that, in the free-space contract system, the carrier provides zero free-time-limit for the customer. Hence, the customers need to pay the storage fee from the beginning of the container dwell period. Therefore, customers prefer the free-time contract system.

\section{Conclusion}

In this paper, the ocean carrier's pricing schemes for the inbound container storage in the container terminal are investigated from the perspective of supply chain finance. Besides the container terminal, the customer has another container storage place, that is, the RCY. The ocean carrier faces two different charge contracts from the container terminal: the free-time contract and free-space contract. We propose two models to analyze the ocean carrier's price decisions under these two charge contracts.

In the free-time contract system and free-space contract system, we derive the assumptions under which the objective function of the ocean carrier is concave. With these assumptions, we propose the solution approaches for the optimal price schemes. It is shown that, in the free-space contract system, the optimal free-time-limit value in the ocean carrier's price scheme is zero.

The numerical studies compare the two models and show that the carrier is more flexible in the free-space contract system and receives more profit by using the free-storage-space as a pooling storage system. It is found that the free-space contract system benefits both the carrier in profit and the busy terminal in traffic control.

Although we made some assumptions in the model basic settings to let the problem be tractable, the results and insight achieved in this paper may provide a valuable managerial guide for the ocean carriers. We also raise, for the first time, the pricing scheme problem in the unaligned ocean transportation system, which provides impetus for future research.

A possible extension about this paper is the research concerning the three-tier contract system which involves both the contract between the container terminal and the ocean carrier and the contract between the ocean carrier and the customer. Moreover, the priority pricing scheme and stochastic container arrival pattern could also be future research directions.

\section{Conflict of Interests}

The authors declare that there is no conflict of interests regarding the publication of this paper.

\section{Acknowledgments}

The authors would like to express their sincere appreciation to the three independent referees in making valuable comments and constructive suggestions to the paper. Their comments have improved the quality of the paper immensely. The work described in this paper was fully supported by the National Science Fund for Distinguished Young Scholars (Grant no. 71025005), the National Natural Science Foundation of China (Grants nos. 71302109, 71202114, and 91224001), and the President Fund of UCAS (Y35101KY00).

\section{References}

[1] J. C. Fransoo and C.-Y. Lee, "Ocean container transport: an underestimated and critical link in global supply chain performance," Production and Operations Management, 2010.

[2] H.-O. Günther and K.-H. Kim, "Container terminals and terminal operations," OR Spectrum, vol. 28, no. 4, pp. 437-445, 2006.

[3] R. Stahlbock and S. Voß, "Operations research at container terminals: a literature update," OR Spectrum, vol. 30, no. 1, pp. $1-52,2008$.

[4] B. de Castilho and C. F. Daganzo, "Optimal pricing policies for temporary storage at ports," Transportation Research Record, vol. 1313, pp. 66-74, 1991.

[5] K. H. Kim and K. Y. Kim, "Optimal price schedules for storage of inbound containers," Transportation Research B: Methodological, vol. 41, no. 8, pp. 892-905, 2007.

[6] C.-Y. Lee and M. Yu, "Inbound container storage price competition between the container terminal and a remote container 
yard," Flexible Services and Manufacturing Journal, vol. 24, no. 3, pp. 320-348, 2012.

[7] J. Holguín-Veras and S. Jara-Díaz, "Optimal pricing for priority service and space allocation in container ports," Transportation Research B: Methodological, vol. 33, no. 2, pp. 81-106, 1998.

[8] J. Holguín-Veras and S. Jara-Díaz, "Preliminary insights into optimal pricing and space allocation at intermodal terminals with elastic arrivals and capacity constraint," Networks and Spatial Economics, vol. 6, no. 1, pp. 25-38, 2006.

[9] S. Saurí, J. Serra, and E. Martín, "Evaluating pricing strategies for storage in import container terminals," Transportation Research Record, no. 2238, pp. 1-7, 2011.

[10] I. Watanabe, Container Terminal Planning: A Theoretical Approach, World Cargo News (WCN), Great Britain, UK, 2001.

[11] C.-Y. Lee and M. Yu, "Inbound Container Storage Price Competition between the Container Terminal and a Remote Yard," Flexible Services and Manufacturing Journal, vol. 24, no. 3, pp. 320-348, 2012. 


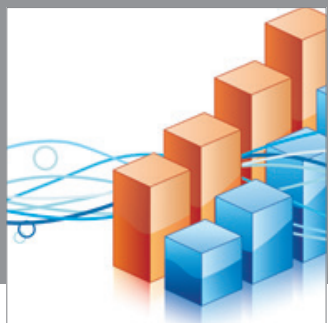

Advances in

Operations Research

mansans

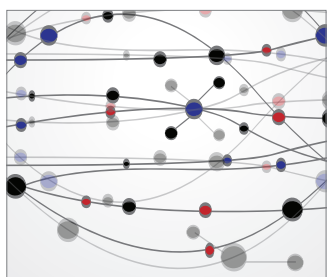

The Scientific World Journal
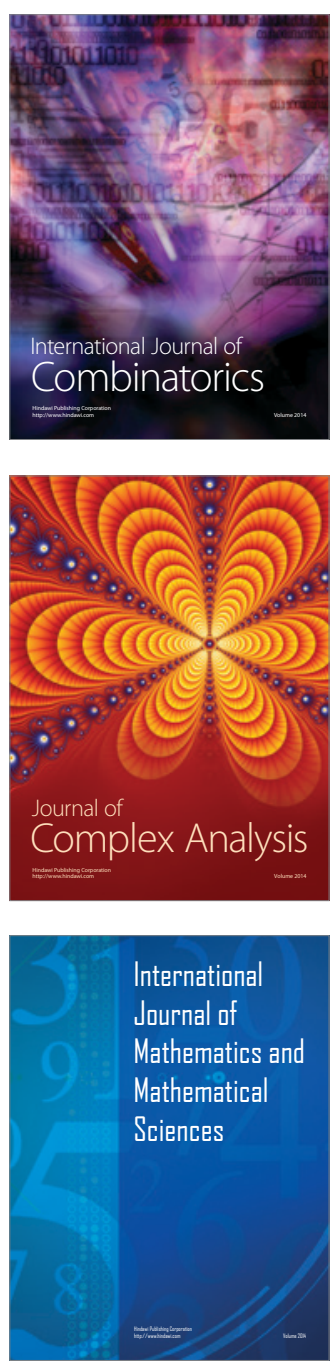
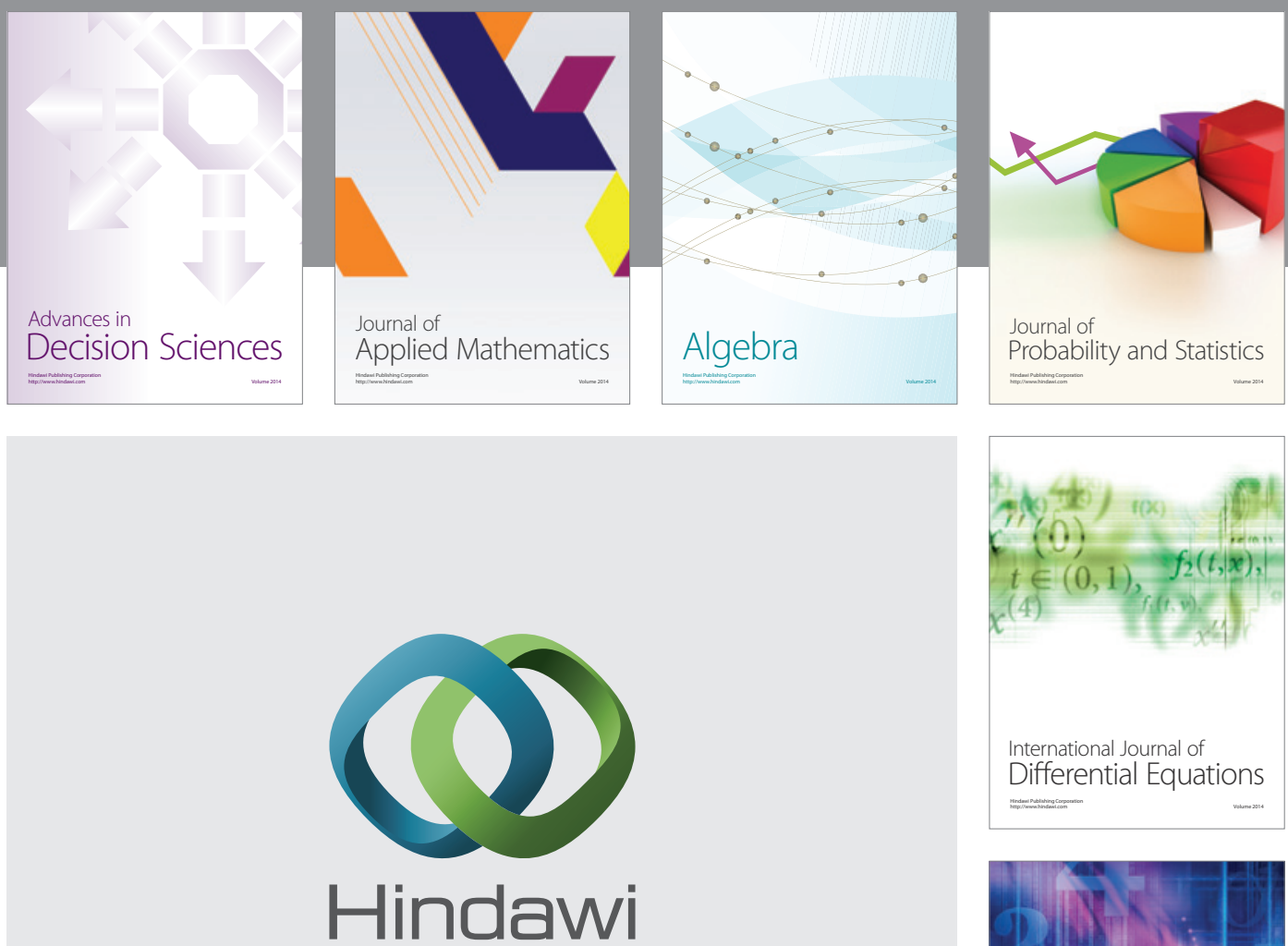

Submit your manuscripts at http://www.hindawi.com
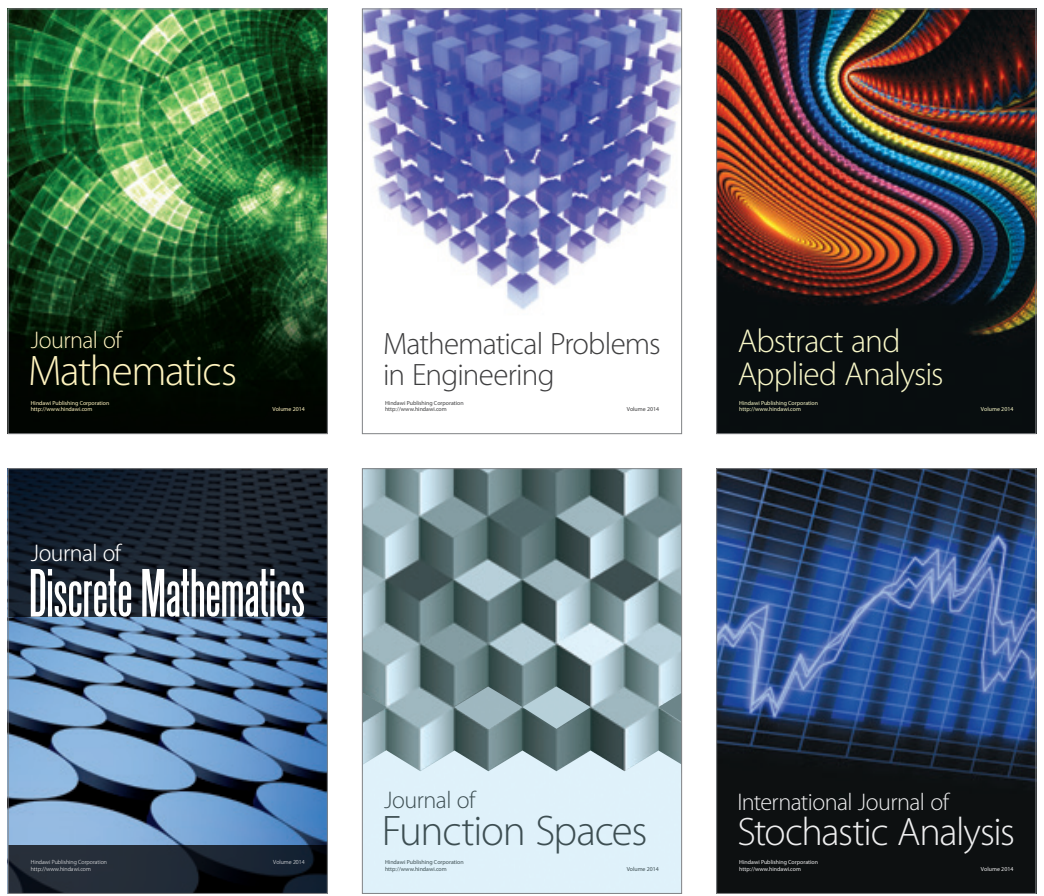

Journal of

Function Spaces

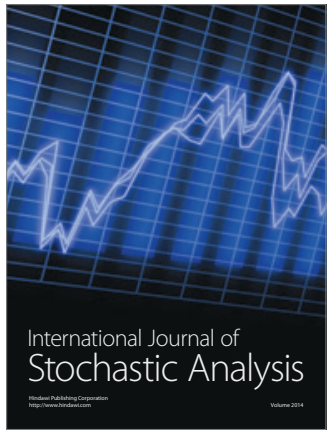

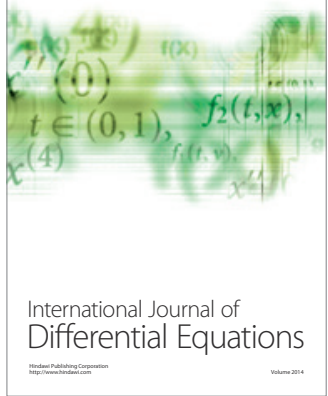
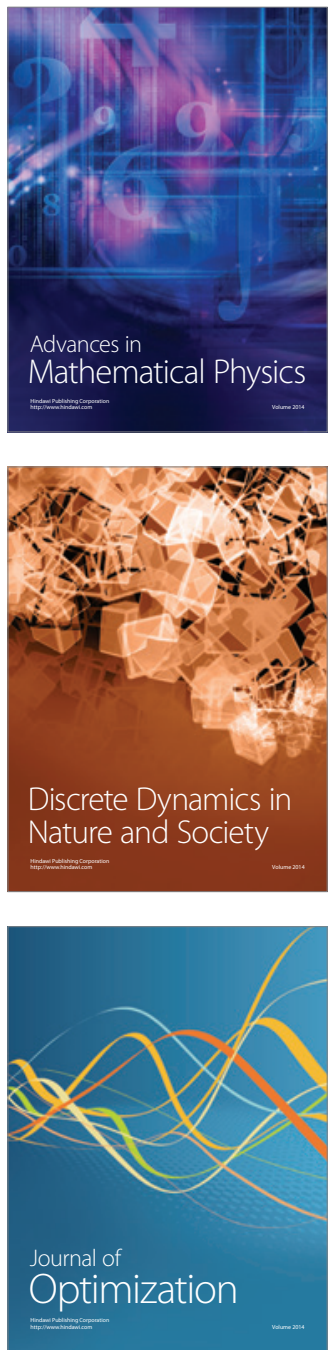